\title{
Death and Injury Due to All-Terrain Vehicles - A Disturbing Trend
}

Can. J. Neurol. Sci. 2010; 37: 301

The use of all-terrain vehicles (ATVs) among Canadians continues to increase. For some, they are an enjoyable way to explore our country's vast network of recreational trails, for others a valuable tool for farming or ranching, and for those in remote communities, an often indispensable part of day-to-day life. No matter what the reason for their use, an evolving literature continues to highlight that ATVs can be an extremely dangerous and often lethal mode of transportation.

In this issue of the Canadian Journal of Neurological Sciences, Lord, Tator and Wells examine deaths due to ATV use in Ontario in a ten-year period from 1996-2005 ${ }^{1}$. Their results are disturbing and should serve as a call to action to physicians with an interest in injury prevention - especially those in the clinical neuroscience community.

As their use increases across the country, so do the number of injuries resulting from ATV use with a $50 \%$ increase in hospitalizations in the five years ending in 2001. Relative to cycling accidents, ATV injuries are more severe, more likely to require operative intervention and associated with a longer length of stay $^{2}$. Their frequency is also increasing at a faster rate than cycling injuries $^{3}$. Warda has shown that helmet use among ATV riding children is low, and dangerous riding habits common ${ }^{4}$.

While a total of 74 ATV-related occurred in the study period, most of which were related to severe head injury, Lord also reveals several factors of even more concern: the number of annual deaths increased dramatically over the ten years studied, more than one fifth of the deaths occurred in children under 16, alcohol was a factor in almost $50 \%$ of fatalities and more than $60 \%$ of deaths occurred in persons not wearing a helmet. In addition, there is an overwhelming male predominance in ATVrelated deaths- $90.5 \%$ were men. Although the study was confined to Ontario, Sibley and Tallon showed almost identical demographics in a similar study of ATV-related injuries in Nova Scotia. While concerning, these trends also give us insight into where to focus our efforts to ensure that these worrisome statistics do not worsen. Through a combination of education, legislation, advocacy and design improvements, reversal of these trends is both a realistic goal and a public health imperative.

Education and public awareness of the importance of safe riding and helmet use has been shown to decrease risky behaviours and injuries in children ${ }^{5}$. The importance of helmet use and safe ATV riding must be stressed by the ATV industry and its dealers and the injury prevention community, including neurosurgeons, neurologists, pediatricians and primary care physicians.

Legislation regulating ATV use varies widely across the country $^{6}$. Some provinces have minimal requirements of ATV riders, with no age-restriction or need for helmet use, while others require drivers to be a minimum of 16 years-of-age, wear a helmet and take a safety course. Safety-related legislation for ATVs can dramatically reduce injury rates ${ }^{7}$. Effective legislation should include a minimum age for drivers of 16, mandatory helmet use, and some form of driver certification.

Various groups, including the Canadian Pediatric Society ${ }^{6}$ and the Canadian Association of Pediatric Surgeons ${ }^{8}$ have put out position statements on the use of all-terrain vehicles by children. ThinkFirst Canada and its chapters across the country, through its funding of injury prevention research such as Lord's study, as well as its strong injury prevention message in schools and the media continues to play a major role. Advocacy such as this, by individual physicians or specialty societies serves to educate the public and pressure governments to act to improve safety. Given the significant number of brain and spinal injuries associated with ATV use, the Canadian Neurological Sciences Federation and its member organizations can and should play an important role in advocating for safer use of ATVs.

Manufacturers of ATVs must also play a role. In response to an unacceptable risk of rollover, three-wheeled ATVs are no longer manufactured. The ATV industry must continue to develop and implement safety features to help make the risk of injury as low as possible.

The neuroscience community in Canada, in large measure led by the Herculean efforts of Charles Tator through his founding and leadership of ThinkFirst Canada, has already made demonstrable inroads in reducing serious head and spine injury among Canadians. This study underscores the need to continue to expand our efforts through education, legislation and advocacy to prevent ATV-related injuries.

Patrick McDonald

Winnipeg, Manitoba, Canada

\section{REFERENCES}

1. Lord S, Tator C, Wells S. Examining Ontario deaths due to allterrain vehicles, and targets for prevention. Can J Neurol Sci. 2010;37:343-9.

2. Helmkamp JC, Aitken ME, Lawrence BA. ATV and bicycle deaths and associated costs in the United States, 2000-2005. Public Health Rep. 2009;124(3):409-18.

3. Brown RL, Koepplinger ME, Mehlman CT, Gittelman M, Garcia VF. All-terrain vehicle and bicycle crashes in children: epidemiology and comparison of injury severity. J Pediatr Surg. 2002;37(3):375-80.

4. Warda L, Klassen TP, Buchan N, Zierler A. All terrain vehicle ownership, use, and self reported safety behaviours in rural children. Inj Prev. 1998;4(1):44-9.

5. Vassilyadi M, Duquette C, Shamji MF, Orders S, Dagenais S. Evaluation of ThinkFirst for kids injury prevention curriculum for grades 7/8. Can J Neurol Sci. 2009;36(6):761-8.

6. Preventing injuries from all-terrain vehicles. Paediatr Child Health. 2004;9(5):337-46.

7. Fonseca AH, Ochsner MG, Bromberg WJ, Gantt D. All-terrain vehicle injuries: are they dangerous? A 6-year experience at a level I trauma center after legislative regulations expired. Am Surg. 2005;71(11):937-40; discussion 940-1.

8. Trauma Committee of the Canadian Association of Pediatric Surgeons. Canadian Association of Pediatric Surgeons' position statement on the use of all-terrain vehicles by children and youth. J Pediatr Surg. 2008. 43(5):938-9. 